\title{
LAS DOS CARAS DE LA MODERNIDAD: HEIDEGGER Y SCHMITT
}

\author{
The two faces of modernity: Heidegger and Schmitt
}

José Fernando García*

\section{RESUMEN}

El artículo coteja las posiciones de Heidegger y Schmitt sobre la modernidad, tomando como referencia La época de la imagen del mundo y El Leviathan en la teoría del Estado de Thomas Hobbes -textos originados en respectivas conferencias dictadas en 1938- en las que ambos pensadores caracterizan como época de la técnica. El contrapunto con Schmitt permite mostrar la unilateralidad del análisis de Heidegger, dado que ignora lo que este destaca, el surgimiento de la distinción privado-público que la subjetividad hace posible. El planteamiento de Heidegger, a su vez, hace ver, contra Schmitt, que dicha distinción no es una "falla" en el argumento de Hobbes sino que está presupuesta en su punto de partida. E igualmente, que la decisión política que Schmitt contrapone a la neutralización del Estado mediante la ley deriva asimismo de la subjetividad, con lo cual el intento de relegar la segunda en favor de la primera se revela insostenible.

Palabras clave: Época Moderna, subjetividad, Estado, técnica, decisión, neutralidad, Heidegger, Schmitt.

\section{ABSTRACT}

The article compares the positions of Heidegger and Schmitt on modernity, with reference to The age of the world picture and The Leviathan in the State Theory of Thomas Hobbes-texts

\footnotetext{
* Universidad Academia de Humanismo Cristiano. Santiago, Chile. Correo electrónico: jgarcias@ docentes.academia.cl
}

Artículo recibido el 24 de octubre de 2013. Aceptado el 22 de julio de 2014. 
originating in respective lectures given in 1938-, in which both of them characterize it as the epoch of technique. The counterpoint with Schmitt displays the sidedness of Heidegger's analysis, as it ignores what that stands, the emergence of private-public distinction that subjectivity makes possible. Heidegger's approach, in turn, makes it manifest, against Schmitt, that this distinction is not a "failure" in Hobbes's argument but is presupposed as its starting point. And too, that the political decision which Schmitt counter pose with the neutralization of the State by the law is also derived from subjectivity, with which the attempt to relegate it in favor of the first is revealed as unsustainable.

Keywords: Modern Epoch, subjectivity, State, technique, decision, neutrality, Heidegger, Schmitt.

El año 1938, Carl Schmitt pronunció dos conferencias (en Leipzig, el 21 de enero y en Kiel el 29 de abril) sobre El Leviathan en la Teoría del Estado de Thomas Hobbes. El 9 de julio del mismo año, Martin Heidegger dictó en Friburgo La fundamentación de la moderna imagen del mundo por medio de la metafísica (Schmitt, 1990; Heidegger, 1995). En dichas conferencias ambos pensadores caracterizan a la Época Moderna como la época de la técnica mecanizada, con matices distintos, como veremos, enfocándose Schmitt en el Estado y Heidegger en la ciencia, en los dos casos con tonos sombríos.

El planteamiento de Heidegger sobre la ciencia y la tecnología ha tenido gran influencia en el pensamiento contemporáneo y es afín a la preocupación de pensadores pertenecientes a distintas tradiciones por el poder y la autonomía de la tecnología en nuestra actualidad (I). La posición de Schmitt se presta a mayor controversia, puesto que toma una posición negativa frente al Estado de derecho y al Estado constitucional, de amplia legitimidad en el mundo contemporáneo. No obstante, su análisis expone con insuperable lucidez el germen de esas nociones en la subjetividad, donde Heidegger hace radicar igualmente la condición destructiva de la ciencia y de la técnica. El contrapunto con Schmitt permite mostrar la unilateralidad del análisis de Heidegger, dado que ignora lo que aquel bien exhibe, por cierto, deplorándolo: la contracara de la modernidad en la distinción privadopúblico que la subjetividad hace posible y la destrucción del Leviathan -del Estado Total-, merced a los "poderes de la sociedad" a que esta da lugar (II). El análisis de la subjetividad efectuado por Heidegger permite mostrar, a su vez, contra Schmitt, que la distinción fuero interno-fuero externo no es una "falla" en el argumento de Hobbes sino que está presupuesta en su punto de partida (III). Igualmente, que la noción de "decisión", que contrapone a la "neutralización" moderna del Estado, deriva como ésta de la subjetividad, con lo cual su intento de relegar la segunda en favor de la primera se revela insostenible (IV). 
Para Heidegger uno de los fenómenos esenciales de la Edad Moderna es la ciencia y esta se distingue de la ḱ se convierte en investigación, lo cual supone: 1) la apertura de una región de los entes de acuerdo a la proyección de un rasgo fundamental (el carácter de cuerpo de los cuerpos, lo que las plantas tienen de plantas, lo animal de los animales); 2) la anticipación, esto es, la constitución por adelantado de algo como ya conocido (en la física, movimiento significa cambio de lugar, ningún movimiento ni dirección del movimiento destaca respecto al resto, todo lugar es igual a los demás, no hay ningún punto temporal que tenga supremacía, la fuerza se determina como magnitud del cambio de lugar por unidad de tiempo); 3) la vinculación de 1) y 2) que determina el rigor como exactitud; 4) el método, mediante el cual la anticipación representa lo variable en su transformación a través de reglas, leyes y experimentos; 5) el carácter de empresa, el regirse por los propios resultados y disposiciones que permiten el control y la planificación.

La pregunta de Heidegger es ¿qué concepción del ente y qué concepto de verdad hacen posible que la ciencia se convierta en investigación, es decir, cuál es su fundamento metafísico? Su respuesta: la ciencia llega a ser investigación cuando el ser de los entes se interpreta como objeto y a la verdad como certeza de la representación. Esto ocurre por primera vez en la obra de Descartes y toda la metafísica moderna se mantiene, de acuerdo a él, en dicha interpretación, poniendo las bases para que esta sea teoría del conocimiento. El conocimiento pide cuenta al ente sobre cómo y hasta qué punto está a disposición de la representación, es decir, hasta dónde es objeto. Así, Descartes es el primero en colocarse en la posición de tener que demostrar la realidad del mundo exterior.

El término griego v́токє́́ $\mu \varepsilon v o v$, equivalente al latino subjectum, significa lo que como fundamento reúne todo sobre sí. Con Descartes el hombre se convierte en el primer y auténtico subjectum, lo que quiere decir que es el ente en el que se fundamenta el resto de los entes en cuanto a su modo de ser y a su verdad. La esencia del hombre se transforma desde el momento en que se convierte en sujeto, cuando se muda en centro de referencia del ente como tal. Pero esto es posible en tanto se modifica también la concepción de los demás entes; ahora se entienden de tal modo que son y solo pueden ser en cuanto son puestos por el hombre que representa.

Representar implica situar algo ante sí a partir de sí mismo asegurándolo y solo el cálculo puede lograr garantizar la certeza de aquello que está enfrente. La naturaleza se convierte en objeto de la representación cuando esta consigue calcularlo por adelantado en su transcurso futuro o a posteriori como pasado. El cogito ergo sum, la certeza fundamental es la representación simultánea del representador y lo 
representado. "La certeza fundamental es el me cogitare=me esse indudablemente representable y representado en todo momento" (Heidegger, 1995: 88). En esa certeza el cálculo se asegura como ámbito de toda representatividad del objeto. Con esto el hombre se fundamenta a sí mismo como medida de todos los entes, de todo aquello que puede pasar por cierto, esto es, por verdadero y por algo que es.

El subjetivismo, el mundo como imagen y el humanismo son procesos entretejidos. La conquista del mundo como imagen significa que los entes en su totalidad quedan situados ante el hombre y quedan a su disposición como sistema, derrotero correlativo al humanismo, en el cual el hombre da la medida de todo ente, de las normas y los valores. Descartes produce una inversión del pensamiento griego; a partir de él se busca y encuentra el ser de los entes en su representatividad. Para los griegos, por el contrario, el hombre es en tanto que percibe a los entes, por lo que para ellos no podía haber imagen del mundo ni humanismo.

Heidegger no deja de mencionar que la época moderna puede ser vista correctamente como el tiempo en el que el hombre se libera de las ataduras medievales, pero añade que dicha caracterización es superficial e impide captar el fundamento de dicha época; lo esencial es el juego alternante y necesario entre subjetivismo y objetivismo. Esto se revelaría en el hecho de que ninguna época anterior creó un objetivismo comparable, ni la colectivización e uniformidad humanas adquirieron una importancia equivalente.

Además de esa referencia, la libertad que hace posible la subjetividad cartesiana es un tema ausente en la conferencia La fundamentación de la moderna imagen del mundo por medio de la metafísica. Para Heidegger, el fenómeno fundamental de la época moderna es la ciencia y la técnica vinculada al objetivismo y a la disposición sobre los entes que este implica. "En el imperialismo planetario del hombre técnicamente organizado, el subjetivismo del hombre alcanza su cima más alta, desde la que descenderá a instalarse en el llano de la uniformidad organizada" (Heidegger, 1995: 89).

En sus trabajos posteriores Heidegger introduce otros matices, entendiendo la tecnología como un modo de des-ocultar que desafía (herausfordern) a la naturaleza a poner (stellen) sus productos, que la dispone (bestellen) como recurso (Bestand), cuya esencia se expresa en el término Das Gestell (la armazón). Al mismo tiempo, extrema, si cabe, su posición anti-humanista: "La esencia de la técnica en cuanto este destino, introduce al hombre en lo que él mismo y por sí mismo ni puede inventar ni, mucho menos hacer; pues, algo así como un hombre que sea hombre única y solamente por sí mismo, no lo hay" (Heidegger, 2003: 143). El hombre es libre en tanto pertenece al ámbito del destino y no está ordenado ni a la voluntad ni a la causalidad, sino al des-ocultamiento, a escuchar el advenimiento de la verdad. 
Schmitt comienza sus conferencias sobre El Leviathan reivindicando el carácter de símbolo mítico que tiene la imagen. Como toda imagen, esta es susceptible de múltiples interpretaciones y la cuestión a la que se orienta Schmitt es a dilucidar si el mito del Leviathan creado por Hobbes fue en realidad el restablecimiento de la unidad política originaria de los poderes temporal y espiritual -pagana, lo recuerda-, contra su destrucción judeo-cristiana. La pregunta, agrega más adelante, apunta hacia el mito como fuerza histórica autónoma (Schmitt, 1990: 14 y 28). El subtítulo con que fue publicada la conferencia adelanta la tesis de Schmitt sobre la escasa suerte que tuvo El Leviathan como mito'.

A más del título, lo que ha contribuido, según Schmitt, a la "impresión excepcional" de El Leviathan ha sido el grabado que figura en la primera edición del libro junto al lema tomado del Libro de Job, "ningún poder de la tierra puede compararse con él": un hombre gigantesco, compuesto de innumerables hombres pequeños, tiene en el brazo derecho una espada y en el izquierdo un báculo episcopal que protegen a una ciudad. Debajo de cada brazo, del temporal y del espiritual, figuran cinco dibujos que representan los instrumentos de poder de cada uno de ellos.

Sin embargo, el lector que quiera desentrañar el significado de la imagen a través de la lectura del libro quedará desilusionado, Hobbes es más bien mezquino al respecto. Schmitt cita un pequeño escrito donde este sostiene que el título adecuado sería Behemoth contra Leviathan. El primero también un monstruo bíblico que figura en el Libro de Job y se distingue del segundo por ser terrestre. Behemoth es la rúbrica con la que Hobbes quiso representar la revolución presbiteriana y puritana de 1640 a 1680, como símbolo de la anarquía producida por el fanatismo. Sostiene Schmitt: "El estado es para Hobbes evitación permanente de la guerra civil gracias a un poder gigantesco. De ahí que uno de los dos monstruos, el Leviathan, 'el Estado', sojuzgue siempre al otro, el 'Behemoth', la Revolución” (Schmitt, 1990: 21).

Bajo el nombre de Leviathan Hobbes desliza tres significados, 1) la referencia al monstruo marino de origen bíblico, 2) producto de una construcción jurídica, el contrato, 3) consistente en una máquina dominada por una persona soberana, una máquina con alma. Esta última es para Schmitt la médula de la construcción hobbesiana, en la cual transporta la concepción cartesiana del hombre al "hombre magno", al Estado como una máquina animada. La aguda distinción entre "mecanismo" y "organismo" no se impone sino a fines del siglo XVIII. De ahí que para Hobbes mecanismo, organismo y obra de arte, están implícitas en la máquina

\footnotetext{
${ }^{1}$ La publicación original del texto de Schmitt tiene como subtítulo Significado y fracaso de un símbolo político, no incluido en la edición castellana.
} 
como producto de la energía creadora del hombre, lo que le permite unirla a la imagen del Leviathan.

Pero ni la imagen mítica del Leviathan ni el soberano que la anima pueden cambiar el hecho de que el Estado es para Hobbes fundamentalmente una "gran máquina". Un primer paso se dio, en un siglo hastiado de querellas religiosas, inicialmente recurriendo a un campo neutral en la metafísica natural cuyos principios y conceptos resultaban claros a los ojos de todos y se afirmaban en la evidencia y certeza de las matemáticas. Pero ya con Hobbes importa que las leyes del Estado sean independientes de toda verdad y rectitud substancial de orden religioso o jurídico y que su vigencia se derive exclusivamente de la decisión estatal. Hobbes no distingue ya entre Auctoritas y Potestas: "Auctoritas, non Veritas facit Legem". El resultado es que la máquina se independiza de todo objetivo y convicción política y se presenta frente a los valores y frente a la verdad con la neutralidad propia de un instrumento técnico. Todas las nociones de verdad y de justicia quedan absorbidas en la decisión del mandato de la ley, dado que llevarlas al plano de la argumentación jurídica implicaría suscitar nuevas disputas y, por tanto, inseguridad. La máquina estatal funciona o no, y si lo hace garantiza la seguridad de todos y a cambio exige obediencia incondicional a la ley. Hobbes es el primero en dar expresión conceptual a la idea de Estado como un artificio técnico fabricado por los hombres que halla su "derecho" y su "verdad" en su propio funcionamiento.

Pero, a más de eso, hay en el Leviathan otro giro de mayores consecuencias, un "punto vulnerable", donde "Hobbes falla de pronto al llegar al punto decisivo" (Schmitt, 1990: 54), la brecha donde se origina la posterior transformación del estado de policía en estado de derecho y la consiguiente transformación de la ley en un medio técnico para someter a cálculo al poder estatal.

Según Schmitt, el problema de la fe y de los milagros fue fatal para el Leviathan. Para este nada es verdadero, todo es mandato y, por tanto, milagroso es aquello que el soberano como Vicario de Dios manda creer como tal y deja de serlo cuando este lo prohíbe. El Estado como public reason decide si un hecho debe ser considerado milagro o no. Schmitt recuerda la relevancia que el problema tenía en la época de Hobbes, las curas milagrosas habían sido algo fundamental en el orden monárquico y había que defenderlas en la lucha con el Papa. La cuestión tenía una dimensión política práctica, de ninguna manera meramente teórica. La posición de Hobbes es agnóstica y su crítica frente a los milagros "tiene ya el tono de la Ilustración" (Schmitt, 1990: 53), pero en cuanto que sea milagro es algo sujeto a controversia y potencialmente motivo de división, se requiere la decisión estatal.

No obstante, en este punto culminante del decisionismo y del poder soberano creador de la unidad política y religiosa, Hobbes establece algunas "reservas individualistas indesarraigables, de tal suerte, que hasta huelga plantear siquiera el problema de si Hobbes era o no realmente lo que se llama un 'individualista'" 
(Schmitt, 1990: 54-55). Asume la distinción entre fuero interno y externo, de tal suerte que el problema de los milagros queda entregado a la razón pública pero en virtud de la libertad de pensamiento -quia cogitatio omnis liberta est- es encomendado al fuero de cada cual creer íntimamente o no y conservar en su corazón -intra pectus suum-su propio juicio.

La distinción hobbesiana privado-público ha calado tan hondo, dice Schmitt, que de ella se han derivado en el curso de los siglos posteriores el Estado de derecho y el Estado constitucional. Ambos arrancan de dos elementos que se refuerzan mutuamente: en primer lugar, de la libertad individual de pensamiento y de conciencia construida en sentido jurídico; en segundo lugar, del agnosticismo que justifica un poder puramente externo.

Sólo unos ańos más tarde, la "mirada del primer judío liberal" (Schmitt, 1990: 56) logró descubrir la falla apenas visible en el Leviathan de Hobbes. Spinoza en su Tratado teológico-político publicado en 1670 defiende ya no sólo el principio general de la libertad de pensamiento sino el derecho a manifestarlo. El paso entre Hobbes y Spinoza -que Schmitt tanto lamenta- es efectivamente crucial. El capítulo XX de dicho tratado tiene el siguiente contenido, según el índice: "Se establece que en un estado libre cada cual tiene el derecho de pensar lo que quiera y de decir lo que él piensa" (Spinoza: 1986). En el capítulo XXXVII de El Leviathan, dice Hobbes, por el contrario:

Un hombre particular tiene siempre la libertad (porque el pensar es libre) de creer o no creer en su corazón aquellos hechos que han sido considerados milagrosos, de acuerdo con su estimación del beneficio que puede aportar la credulidad de los hombres a aquellos que lo pretenden o sancionan, y con ello conjeturar si son milagros o mentiras. Pero cuando se trata de confesar aquella fe, la razón privada debe someterse a la pública, es decir, al lugarteniente de Dios (Hobbes, 1980: 506).

Schmitt es enfático, lo que llevó al Dios mortal a la muerte fue la distinción interno-externo. El Estado, la Ley y el Derecho quedan transformados: el primero es privado de su alma -de su capacidad de decisión, debemos entender-, convirtiéndose en una simple máquina; la segunda, en un medio técnico para enfrentar al Estado, y el rasgo característico de todo el proceso es la trasformación de la legitimidad en legalidad, del derecho en derecho positivo. El Estado Absoluto del siglo XVIII es desplazado por el Estado de Derecho Burgués del siglo XIX y su precursor espiritual es Hobbes en un doble sentido. Desplazó, por una parte, las nociones medievales del derecho divino de los reyes y, en general, los conceptos del derecho entendidos en sentido substancial, por otra, introdujo las "reservas" individualistas sobre la libertad de conciencia. De ambos desplazamientos se deriva el Estado de derecho fundado, en último término, en el acuerdo de una asamblea constituyente. 
El dualismo estado-sociedad a la que da lugar la distinción entre fuero interno y externo, se traduce en la proliferación de poderes indirectos, tales como partidos políticos, sindicatos, iglesias, etc., que a través del parlamento logran apoderarse del Estado legal. Por "poderes indirectos" Schmitt se refiere a aquellos poderes que exigen obediencia sin ser capaces de proteger. Hay una conexión "eterna" entre protección y obediencia, lo que implica asumir el peligro de lo político responsablemente (Schmitt, 1990: 83). Esa es la significación profunda del Leviathan, intentar poner término a la anarquía del derecho a resistencia feudal, canónica y estamental, oponer a los poderes indirectos la unidad de un poder inequívoco. Pero ni la imagen mítica ni el alma del Leviathan pueden sobrevivir, en definitiva, a la época de la técnica total y a los cambios planetarios a que da lugar. "Esta se considera a sí misma lo suficientemente fuerte para acogerlo bajo su protección, como a los otros saurios y mastodontes, y para mostrarlo en el jardín zoológico como un objeto de museo" (Schmitt, 1990: 82).

\section{III}

Comencemos por preguntar ¿Hobbes "falla al llegar al punto decisivo"? ¿La "falla" no está puesta desde las primeras líneas de El Leviathan? ¿Es meramente "un punto vulnerable"? ¿Podría sostenerse su argumentación toda sin la distinción interno-externo?

La primera parte del libro lleva por título El Hombre, que es la materia y artífice del Leviathan, según nos dice Hobbes en la Introducción. El capítulo primero, Del sentido, comienza con las siguientes palabras:

En cuanto a los pensamientos del hombre, los consideraré primero singularmente, y luego en secuencia ordenada o en su mutua dependencia. Singularmente, son todos una representación o apariencia de alguna cualidad o de otro accidente de algún cuerpo ajeno a nosotros, esto es, de aquello generalmente llamado objeto (Hobbes, 1980: 123).

Claramente, Hobbes pone desde el comienzo su análisis en el terreno de la subjetividad, esto es, en la distinción fuero interno y fuero externo, entendiendo que la relación entre ambos se lleva a cabo a través de la representación. Al igual que Descartes llama pensamiento a todo aquello que se halla en la mente, las sensaciones, la imaginación, la memoria y el entendimiento. Al tiempo, asume decididamente un punto de vista galileano, la vida es movimiento, las cualidades sensibles, - "el arquetipo de todos los pensamientos"- (Hobbes, 1980: 123) son movimientos de la materia que afectan diversamente nuestros órganos. Consecuentemente, rechaza 
la concepción aristotélica -vigente todavía en la época- que sostiene la existencia de species inteligibles, cuya proyección en los sentidos explicaría los fenómenos, aceptando el nominalismo; los universales no son más que nombres, las cosas nombradas individuos.

El vínculo subjetivismo-nominalismo no es contingente, la retirada del mundo que supone el cogito cartesiano -el sujeto desvinculado- es posible en la medida que se ha roto con la idea de un cosmos al cual el hombre estaría atado. Contra Schmitt se puede mostrar que del subjetivismo-nominalismo -asumido desde el inicio por Hobbes- surge el complejo de ideas que moviliza toda la argumentación de El Leviathan.

En primer lugar, la noción de libertad en el sentido moderno de la expresión; el hombre ya no está atado a una razón cósmica, que todo orden tendría que replicar. Hobbes puede dar por el traste con el derecho divino de los reyes y con los derechos estamentales de rebelión frente a la injusticia en cuanto levanta el supuesto de dicha razón cósmica del cual estos serían expresión.

En segundo lugar, la pérdida de vigencia en la creencia en un orden natural que por sí mismo liga a los miembros de una comunidad, implica la disolución de la misma y el surgimiento del individualismo que se expresa en el contractualismo hobbesiano. Es más, si no hay un orden que la naturaleza tenga seguir, si esta carece de un propósito intrínseco, es posible pensar en el mecanicismo de la misma, asumida por Hobbes. A su vez, esto tiene la implicación de que en la medida que la naturaleza no puede ya dictar una idea de bien a la cual subordinarse, esta tiene que tener su fuente en el consentimiento de la voluntad (Taylor, 1989).

El subjetivismo-nominalismo es también una condición sin la cual no habría sido posible la ciencia moderna. Sobre la idea de unas species propias de la realidad no podría haber ciencia empírico-matemática. Una de las características de la Nueva Ciencia propuesta por Galileo es limitarse a investigar cómo los fenómenos se relacionan entre sí, argumentando la futilidad de pretender conocer dichas species. El supuesto galileano que funda la ciencia moderna es que la naturaleza está escrita en un lenguaje matemático y eso implica una ontología inseparable del nominalismo, básicamente la homogeneidad del espacio y del tiempo, es decir, la ausencia de jerarquía y orden propios de la naturaleza, y la necesidad consiguiente de representarse toda cualidad al modo geométrico.

De modo que la subjetividad y la distinción de fuero interno y externo que implica es el terreno mismo en el cual se planta la teoría de Hobbes. Esto permite mostrar, contra Schmitt, que es mucho más que "un punto vulnerable" en El Leviathan, que este no falla al llegar "al punto decisivo", y si hubiera que expresarse de ese modo se trataría de una "falla geológica" que compromete a toda la construcción hobbesiana. Pero el análisis de Schmitt, con toda su ambigüedad, 
permite arrojar luz sobre aquello que Heidegger desconoce: que la libertad subjetiva como característica de la Época Moderna es mucho más que "correcta" pero "superficial", puesto que ahí se originan, como aquel lo muestra en forma brillante, el Estado de derecho y el Estado constitucional.

Las críticas respectivas de Heidegger y Schmitt permiten reconocer en la subjetividad el origen de lo que podemos llamar las dos caras de la modernidad: por una parte de la ciencia y la técnica como una potencia que tiende a escapar a todo control, por otra, del Estado como producto del consentimiento y cuyo poder debe sujetarse a la ley.

\section{IV}

¿Qué es, en definitiva, lo que Schmitt rescata de El Leviathan de Hobbes? Desde luego, rechaza que el Estado pueda dar ser el resultado de "un contrato entre simples individuos"... "por obra de conceptos analíticos de ese racionalismo" (Schmitt, 1990: 32). El otro elemento que nace del contrato social, único garante de la paz, la persona soberana, no se produce por obra del consentimiento sino con ocasión de dicho consentimiento, sostiene Schmitt. La persona soberana representativa es infinitamente más que la suma de las voluntades de los participantes en el contrato. "En este sentido, el nuevo dios es trascendente a los individuos que han celebrado el contrato y es trascendente también a su suma, pero sólo en sentido jurídico, no en sentido metafísico" (Schmitt, 1990: 33). Hobbes llegó a concebir en el siglo XVII con sorprendente claridad y hasta sus últimas consecuencias la idea de Estado creado por la razón de los individuos. El paso decisivo es la concepción del Estado como producto artificial del cálculo y como una máquina con alma. Con esto dio un paso más allá de Descartes en la interpretación antropológica del hombre. Pero la lógica que entiende el Estado como un artificio fabricado por el cálculo de los hombres, no lleva a la persona sino a la máquina. De ahí que el Estado moderno pueda considerarse el primer producto de la época técnica, no solamente el supuesto histórico y sociológico de la misma, cuyo resultado final es que la persona representativa soberana, "el nuevo dios", no pueda mantenerse y quede reducido a mecanismo.

Schmitt aplaude a Hobbes por haber terminado con los poderes indirectos del feudalismo basados en los derechos estamentales, poniendo la unidad de un poder inequívoco, al tiempo que lamenta la transformación del Estado en un sistema de legalidad ajeno a todo contenido sustantivo de verdad o justicia. Pero ambas cosas son inseparables; Schmitt parece estar argumentando contra los derechos sustantivos estamentales a favor del absolutismo estatal, al tiempo que le critica a este su neutralidad agnóstica, sin la cual lo primero no habría sido posible. 
Lo que Schmitt admira de Hobbes es el decisionismo, haber entendido, tan temprano como el siglo XVII, que el derecho divino de los reyes había fenecido y que eso exigía un poder absoluto -la dictadura- capaz de asumir la decisión que exige la política. Como hemos visto, de acuerdo a Schmitt, Hobbes no hizo sino transportar la concepción cartesiana del hombre como máquina con alma al soberano, al "hombre magno". El problema con el Estado absoluto es que el soberano, a quien pertenece la decisión, termina neutralizado por la ley que protege la libertad individual, con lo que acaba perdiendo su alma. Pero si se trata de un transporte, la capacidad de decidir del soberano tiene que haber radicado originalmente en el hombre "pequeño", como lo llama Schmitt (Schmitt, 1990: 37). Es obvio, por lo demás, que sin la decisión de los individuos no habría contrato. Hemos visto, sin embargo, que Hobbes toma distancia de este como origen del Leviathan. No obstante, aun haciendo abstracción del contrato sigue siendo válido que la figura del traslado implica que aquello trasladado existe con anterioridad al traslado ${ }^{2}$.

La decisión es un concepto tributario de la subjetividad, de la distinción fuero interno y externo y, por tanto, la noción que Schmitt moviliza en contra de la neutralidad del Estado de derecho comparte con este los mismos supuestos. Dicho de otro modo: la neutralización contra la cual se alza Schmitt y la decisión que reivindica son conceptos entretejidos. Ya hemos visto que Heidegger subraya que el cogito cartesiano es cogito me cogitare, la conciencia es esencialmente autoconsciencia de la representación y solo como tal es posible la conciencia. En un curso dictado posteriormente (1940) es más explícito: "Cogito sum' no dice ni solo que yo pienso, ni solo que yo soy, ni que del hecho de mi pensar se sigue mi existencia. La proposición habla de una conexión entre cogito y sum. Dice que soy en cuanto aquel que representa, que no solo mi ser está determinado esencialmente por este representar sino que mi representar, en cuanto repraesentatio determinante, decide sobre la praesentia [Prâsenz] de todo representado, es decir sobre la presencia [Anwesenheit] de lo en él mentado, es decir sobre el ser de este mismo en cuanto ente" (Heidegger, 2000: 135) ${ }^{3}$.

\footnotetext{
${ }^{2}$ Desde un punto de vista pragmático cabe argumentar que el "transporte" de un concepto desde un ámbito a otro cambia su significado, pero eso no podría ser aceptado por Schmitt, dado que le quitaría toda fuerza al principio que "todos los conceptos significativos de la moderna teoría del Estado son conceptos teológicos secularizados", y a su pretensión de que hay una "teología política" (Schmitt, 2001: 43).

${ }^{3}$ Antes ha dicho: "Pero ante todo tenemos que constatar que para Descartes la esencia del representar ha trasladado su peso al re-mitir-se de lo re-presentado, en lo cual el hombre que representa decide de antemano y siempre desde sí qué puede y debe valer como sentado y constante". Y sigue: Aquí se anuncia qué subyace como fundamento, cuál es el subiectum -a saber, el representar-y para qué es subiectum el sujeto -a saber, para la esencia de la verdad-" (Heidegger, 2000: 131). De un modo más categórico: "En el hombre en cuanto subiectum se encuentra ahora la decisión esencial acerca de qué habrá de afirmarse en general como ente” (Heidegger, 2000: 141).
} 
Mi representar decide sobre la presencia de los entes, he ahí el fundamento de la decisión, inseparable de la subjetividad. Para Heidegger eso significa que en la Época Moderna "el hombre es la medida de todas las cosas", pero expresa lo contrario que en la frase de Protágoras, puesto que esta dice, a la inversa, "por medio de la permanencia junto a lo presente, la pertenencia del Yo a lo presente es" (Heidegger, 1995: 85). No es, pues, casualidad que "el que acepta en principio la oposición entre lo interno y lo externo, reconoce de antemano la superioridad de lo interno frente a lo externo" (Schmitt, 1990: 61).

En el mundo griego y posteriormente en el medievo no puede haber decisión, puesto que el hombre es en cuanto es parte de un cosmos. La decisión supone un sujeto desvinculado del cosmos hecha posible por el cogito cartesiano y el nominalismo que lo precedió, los que también dan origen a lo que Schmitt llama "neutralización". No habiendo una razón intrínseca a la realidad, aquella queda como un conjunto de procedimientos neutros que le permiten al sujeto auto-dirigirse para seguir los medios orientados a la obtención de fines diversos. El concepto de método expresa bien el carácter que adquiere la razón en la modernidad. Este consiste en "reglas para la dirección del espíritu" y Descartes lo define como aquellos preceptos que ha de seguir quién no quiera tomar lo falso por verdadero. Derivan de la regla fundamental que es controlar la voluntad por el entendimiento, decidir no aceptar juicios sin tener ideas claras y distintas, esto es, de asegurar la verdad mediante la representación garantizada (Descartes, 1980).

La razón, que en el mundo antiguo supone "la observación de las revoluciones de la inteligencia en el cielo" para "aplicarlas a las de nuestro entendimiento, que les son afines" (Platón, s/f: 22) en Descartes queda como la decisión de la voluntad guiada por el entendimiento, como el control de una facultad del alma por otra.

Poco después, Hobbes lo expresa más directamente: la razón es cálculo, adición y substracción -añade- de las consecuencias de nombres generales convenidos para caracterizar y significar nuestros pensamientos (Hobbes, 1980: 149). El empirismo le impide, sin embargo, aceptar la certeza cartesiana derivada de la existencia de Dios y la consiguiente recuperación del criterio de verdad, consistente en la claridad y distinción de las ideas. Para Hobbes no hay ideas innatas, ni certeza del cálculo y la ciencia tiene, por tanto, un carácter condicional. "Pero ninguna razón de un hombre, ni la razón de ningún número de hombres, asegura la certeza; como tampoco un recuento está bien confeccionado porque muchos hombres lo hayan aprobado unánimemente". Este agnosticismo refuerza la neutralidad y plantea la necesidad de, "a falta de una recta razón constituida por naturaleza”, someterse "a la recta razón de algún árbitro o juez" para que la "controversia no desemboque en puñetazos o quede sin decidir" (Hobbes, 1980: $150)$. 
Mostrada queda, pues, la solidaridad de neutralidad y decisión en cuanto ambas tienen dependencia en el concepto de subjetividad cartesiano. El punto de encuentro de Hobbes y Schmitt es el agnosticismo del primero y el antirracionalismo del segundo. Cada cual piensa que su resultado es el surgimiento del pluralismo, de intereses particularistas, de la controversia y potencialmente de la guerra civil. Pero el intento schmittiano -como el de Hobbes- de optar por la decisión política contra la neutralidad del Estado -la otra cara del pluralismo- es insostenible, dado que ambas forman parte de la misma constelación de ideas y fuerzas históricas de la modernidad, entre las que hay tensión, por cierto, pero en la que ninguna de ellas puede desplazar a la otra.

\section{REFERENCIAS}

Descartes, René. "Reglas para la dirección del espíritu", en Obras Escogidas. Buenos Aires: Editorial Charcas, (1980): 32-131.

Heidegger, Martin. "La época de la imagen del mundo", en Caminos de bosque, Madrid: Alianza Editorial, (1995): 63-90.

Heidegger, Martin. "El nihilismo europeo", en Nietzsche II. Barcelona: Ediciones Destino S. A., (2000): 32-206.

Heidegger, Martin. "La pregunta por la técnica", en Filosofía, ciencia y técnica. Santiago de Chile: Editorial Universitaria, (2003): 113-148.

Hobbes, Thomas. El Leviathan. Madrid: Editora Nacional, 1980.

Platón. Timeo o de la naturaleza. [Edición electrónica]. Universidad de Artes y Ciencias Sociales (ARCIS), www.philosophia.cl, (s/f).

Schmitt, Carl. El Leviathan en la teoria del Estado de Tomás Hobbes. Buenos Aires: Editorial Struhart \& Cía., 1990.

Schmitt, Carl. Der Leviathan in der Staatslehre des Thomas Hobbes. Sinn und Fehlschlag eines politischen Symbols. Kôln: Hohenheim Verlag, 1982.

Schmitt, Carl. "Teología política”, en Carl Schmitt, Teólogo de la política. México: Fondo de Cultura Económica, (2001): 43-62. 
Spinoza, Baruch. Tratado teológico-político. Madrid: Altaya, 1986.

Taylor, Charles. "El yo desvinculado", en Las fuentes del yo. La construcción de la identidad moderna. Barcelona: Paidós, (1989): 159-173. 